\title{
Einflussfaktoren auf die Einstellung zur Frauenquote
}

\author{
Obwohl Frauen sehr gut ausgebildet sind und es seit Jahren gesetzliche Vorgaben zur \\ Gleichstellung gibt, werden erwerbstätige Frauen vielfach noch immer schlechter bezahlt \\ als Männer; sei es, weil sie in Branchen und Berufen arbeiten, für die in Form mittelbarer \\ Diskriminierung ein niedrigeres Entgeltniveau charakteristisch ist, sei es, weil eine unmit- \\ telbare Diskriminierung gegenüber Männern besteht. Auch in Führungspositionen sind \\ Frauen deutlich unterrepräsentiert. Um die vorhandene Kluft zwischen rechtlicher und \\ faktischer Gleichstellung zu reduzieren, haben mehrere europäische Länder Frauenquoten \\ eingeführt. In Deutschland wird darüber aktuell diskutiert und eine Quote in bestimmten \\ Bereichen ist Teil des aktuellen Koalitionsvertrags. Es stellt sich die Frage: Von welchen \\ Faktoren hängt die Einstellung zur Frauenquote in der Bevölkerung ab?
}

FRANZISKA DRESCHER, PETER SCHROTT

\section{Frauen in Führungspositionen}

Bereits seit über 60 Jahren sind Diskriminierungen aufgrund des Geschlechts (und anderer Gruppenzugehörigkeiten) laut Grundgesetz in Deutschland verboten. Ergänzt wurde dies 1994 mit einem Förderauftrag zur tatsächlichen Gleichberechtigung von Frauen und Männern. Die reale Situation sieht jedoch noch immer anders aus. Im Gender Gap Index des Weltwirtschaftsforums (Hausmann et al. 2013), der das Ausmaß der Geschlechterungleichheit angibt, liegt Deutschland zwar auf Platz 14 von insgesamt 136 verzeichneten Ländern, allerdings unter dem Vorzeichen eines „absteigenden Trends", denn 2006, also acht Jahre zuvor, lag Deutschland in der Gesamtwertung immerhin bereits auf dem fünften Platz. Und auch in den diversen Teilbereichen liegt Deutschland deutlich zurück. So ist es im Bereich „Teilnahme am Arbeitsleben und wirtschaftliche Chancen" (Komponenten wie Erwerbstätigen- und Beschäftigungsquoten sowie Entgeltunterschiede) mit Platz 46 nur im Mittelfeld platziert.

Obwohl mittlerweile mehr Mädchen als Jungen das Abitur oder die Fachhochschulreife erlangen (Weinmann 2010) und mehr Frauen als Männer studieren und auch über erfolgreichere Abschlüsse verfügen (Mischke/Wingerter 2012; Destatis 2011), sind Frauen in Führungspositionen und im Top-Management nach wie vor deutlich unterrepräsentiert. So liegt der Frauenanteil in den Aufsichtsräten der Top-200-Unternehmen der deutschen
Wirtschaft bei lediglich $15,1 \%$. Betrachtet man die Vorstandsposten der Top-200-Unternehmen, sinkt der Frauenanteil auf nur noch 4,4\% (Holst/Kirsch 2014). Den höheren Frauenanteil in Aufsichtsräten erklären Holst und Kirsch (ebd.) mit den Mitbestimmungsregelungen der Arbeitnehmerschaft in den Kontrollgremien, die häufiger Frauen entsenden als die Kapitalseite. In den Führungsetagen der privatwirtschaftlichen Unternehmen sind Frauen mit $30 \%$ unter den angestellten Führungskräften vertreten; dieser Prozentsatz hat sich von 2001 auf 2010 um lediglich 3 Prozentpunkte von $27 \%$ auf $30 \%$ erhöht (Holst et al. 2012; Mischke/Wingerter 2012). Laut Sattelberger (2011) ist der Anteil von Frauen im Topmanagement deutscher Großunternehmen in der letzten Dekade lediglich von 5 auf 5,8\% gestiegen. Das Thema erhält jedoch in den letzten Jahren Rückenwind. Einer Studie von Kienbaum (2012) zufolge hatte sich der Anteil von Frauen in Aufsichtsräten der Dax-30-Unternehmen innerhalb der letzten zwei Jahre zwar verdoppelt, die absoluten Zahlen waren aber weiter unbefriedigend (15\%) und der Anteil in den Vorständen lag weiter deutlich darunter (7\%). Trotz deutlicher Medienpräsenz sind die Veränderungen langsam und schwankend, denn in den Vorständen beispielsweise sind die Zahlen aktuell wieder leicht gesunken (5,5\%, vgl. DIW 2014). In den Aufsichtsräten setzte sich der seit $2010 \mathrm{zu}$ beobachtende kontinuierliche Aufwärtstrend fort, sodass aktuell jedes vierte Aufsichtsratsmitglied weiblich ist (24,7\%; DIW 2014). 
Auch verdienen Frauen noch immer deutlich weniger als Männer - bezogen auf Vollzeittätigkeit. Innerhalb der EU liegt das durchschnittliche geschlechtsspezifische Lohngefälle bei $16 \%$ (Europäische Kommission 2012; Mischke/ Wingerter 2012) und der Lohnunterschied zwischen Frauen und Männern in Deutschland beträgt im Durchschnitt sogar 23 \% (unbereinigter Gender Pay Gap, vgl. Mischke/ Wingerter 2012). Ursachen hierfür sind beispielsweise die ungleiche Besetzung von bestimmten Positionen von Frauen und Männern, der größere Anteil von Frauen in Teilzeit oder die unterschiedliche geschlechtsspezifische Berufswahl (Mischke/Wingerter 2012). Selbst in Führungspositionen werden vollzeitbeschäftigte Frauen im Schnitt $21 \%$ schlechter bezahlt als ihre männlichen Pendants (Holst et al. 2012). Ein deutlicher Unterschied ist auch zwischen sogenannten „Männer- und Frauenberufen“ erkennbar. So verdienten Frauen in typischen Frauenberufen 2010 im Schnitt nur 77 \% des Gehalts gleichrangiger Frauen in Männerberufen (ebd.). Bisherige Ansätze zur Verbesserung der Chancengleichheit scheinen demnach zu wenig oder $\mathrm{zu}$ kurz zu greifen.

Um dieses Ungleichgewicht zu relativieren, werden bereits seit den 1960er Jahren diverse Gleichstellungsmaßnahmen diskutiert, welche die Chancengleichheit erhöhen sollen. 2006 trat das Allgemeine Gleichbehandlungsgesetz (AGG) in Kraft, dessen Ziel es laut $\$ 1$ ist, „Benachteiligungen aus Gründen der Rasse oder wegen der ethnischen Herkunft, des Geschlechts, der Religion oder Weltanschauung, einer Behinderung, des Alters oder der sexuellen Identität zu verhindern oder zu beseitigen." In Deutschland beziehen sich solche Maßnahmen v. a. auf Frauen und Menschen mit Behinderung. In letzter Zeit gehen die politischen Bestrebungen vermehrt in Richtung familienpolitischer Maßnahmen, welche die Vereinbarkeit von Beruf und Familie verbessern sollen (z. B. Elterngeld, Ausbau von Kindertagesstätten). Auch wenn die Vereinbarkeit von Beruf und Familie ein wichtiges Element für die Gleichstellung darstellt (vgl. Lindecke 2005), so scheint sie nicht ausreichend, um den Prozentsatz von Frauen in Führungspositionen zu erhöhen.

Die Regierung der Bundesrepublik Deutschland sah hier Handlungsbedarf und diskutierte aktuell diverse Quoten. Während das Familienministerium für eine gesetzliche Pflicht zur Selbstverpflichtung hinsichtlich einer flexiblen Quote in den obersten Führungsebenen aller börsennotierten Unternehmen plädierte, die von jedem Unternehmen selbst festgelegt und anschließend veröffentlicht werden sollte (BMFSFJ 2012a) und hierfür von Bundeskanzlerin Angela Merkel den Rücken gestärkt bekam (z. B. Bundesregierung 2011), setzte sich das Arbeitsministerium für eine verbindliche gesetzlich geregelte Quote von $30 \%$ für DaxUnternehmen ein (z. B. DAPD 2011). Der Koalitionsvertrag sowie ein entsprechender Gesetzesentwurf sehen eine fixe Quote von 30 \% in neu zu besetzenden Aufsichtsräten börsennotierter und mitbestimmungspflichtiger Unternehmen ab 2016 vor (BMFSFJ 2014). Ab 2015 sollen zusätzlich börsennotierte oder mitbestimmungspflichtige
Unternehmen „verbindliche Zielgrößen für die Erhöhung des Frauenanteils im Aufsichtsrat, Vorstand und in den obersten Management-Ebenen " festlegen und veröffentlichen (ebd., S. 6).

In Norwegen, Europas Vorreiter in Sachen Frauenanteil im Aufsichtsrat, müssen seit 2008 mindestens $40 \%$ der Aufsichtsratsmitglieder börsennotierter Unternehmen weiblich sein. Mit diesem Prozentsatz liegt Norwegen weltweit auf Platz eins, gefolgt von Schweden mit bereits nur noch $20 \%$ (König 2010). Inzwischen haben mehrere weitere EU-Länder wie beispielsweise Frankreich, Italien und die Niederlande eine derartige Quote verabschiedet (Storvik/Teigen 2010; Endres 2010; Französische Botschaft Berlin 2011; Germany Trade and Invest 2012; SPD 2012), doch liegen diese oft deutlich unter den politisch geforderten Prozentsätzen. Die damalige EU-Justizkommisarin Reding forderte 2012 eine europaweite Frauenquote für Aufsichtsräte, scheiterte damit allerdings (FAZ vom 23.10.2012). Mittlerweile stimmte das EU-Parlament für eine verbindliche Frauenquote in Aufsichtsräten börsennotierter Unternehmen von $40 \%$ bis zum Jahr 2020 (Europäisches Parlament 2013). Derzeit wird der Vorschlag im Rat der EU diskutiert.

In Deutschland haben Unternehmen mittlerweile die Initiative ergriffen. So hat sich beispielsweise die Deutsche Telekom AG im März 2010 als erstes der Dax-30-Unternehmen öffentlich auf eine Frauenquote im Führungskräftebereich verpflichtet; $30 \%$ bis zum Jahr 2015 (Sattelberger 2011). Sattelberger bezeichnete dies als Resultat der bitteren Erkenntnis, dass beim Thema Gleichberechtigung der Geschlechter, insbesondere im Führungsbereich „große Rhetorik für große Vorhaben, vordergründige Verneigung vor der großen Überzeugung, Auszeichnungen für Aktivitäten ohne entsprechende Resultate, hinterrücks stille Missachtung, Vergessen, unbewusstes Ausblenden, subtile Sabotage oder Akzeptanz der Nichtveränderung" an der Tagesordnung seien (ebd., S.429). In Kooperation mit und auf Anregung des Bundesfamilienministeriums sagten im März 2011 die Dax-30-Unternehmen erstmals die Festlegung individueller Selbstverpflichtungen zur Steigerung des Frauenanteils in Führungspositionen zu (BMFSFJ 2012b). Auch für ihre Aufsichtsräte haben die meisten DAX-30-Unternehmen auf Basis der Vielfaltsempfehlung im Deutschen Corporate Governance Kodex (2012) individuelle Ziele für den Frauenanteil festgelegt (BMFSFJ 2012c). Die Ziele sind seit August 2012 öffentlich einsehbar. Dabei streben 14 Unternehmen einen Frauenanteil von $\geq 20 \%$ und 7 von $\geq 30 \%$ an (ebd.).

\section{Potenzielle Einflussfaktoren auf die Einstellung zur Frauenquote}

Neben juristischen Implikationen, die Gleichstellungsmaßnahmen haben können (vgl. Tomasson et al. 1996; 
Wagner/Schmermund 2000), stellt sich demnach die Frage nach der gesellschaftlichen Akzeptanz. Wagner und Schmermund (ebd.) konnten für verschiedene Gleichstellungsmaßnahmen zeigen, dass eine Maßnahme umso eher akzeptiert wird, je stärker Leistungsmerkmale der sich bewerbenden Person berücksichtigt werden und je höher die Fairness der Maßnahme beurteilt wird. Konsistent zeigt sich auch eine unterschiedliche Akzeptanz von Gleichstellungsmaßnahmen bei den Geschlechtern. Frauen stehen Gleichstellungsmaßnahmen positiver gegenüber (Wagner/ Schmermund 2000; Krings et al. 2007; Schlegel 2004; Wippermann 2010). So sprechen sich auch in der im Folgenden zu diskutierenden Studie (vgl. 3.1) 34\% der Frauen und nur $14 \%$ der Männer für eine Frauenquote aus. Dieser Unterschied ist nicht verwunderlich. Frauen sind sowohl die Betroffenen der aktuell unausgewogenen Situation als auch die Profitierenden einer Quote. Es stellt sich jedoch die Frage, warum viele Frauen gegen und manche Männer für eine Quote sind. Ziel der hier vorzustellenden Analyse ist es, neben dem Geschlecht (Hypothese 1) weitere interindividuelle Einflussfaktoren sowie deren Interaktion mit dem Geschlecht zu identifizieren (Abbildung 1). Die Abschnitte 2.1 - 2.4 skizzieren entsprechende Einflussfaktoren, die anschließend empirisch überprüft (3) und diskutiert (4) werden.

\subsection{Erfahrung im Berufsleben}

Personen, die noch nicht im Berufsleben angekommen sind, haben aller Wahrscheinlichkeit nach in ihrem bisherigen Lebenslauf weniger persönliche Erfahrungen mit Chancenungleichheit gemacht. In Schule und Studium sind die Bedingungen für beide Geschlechter heutzutage in der Regel weitgehend homogen. Üblicherweise stellen sich erst im Berufsleben direkte oder indirekte Erfahrungen mit ungleichen Job- und Karrierechancen ein. Das bekannte Phänomen, an die sogenannte „gläserne Decke“ zu stoßen, ist eng an die Berufswelt bzw. berufliche Karrierewege gebunden. Somit ist zu erwarten, dass Personen, die mit ungleichen Rahmenbedingungen in der Berufswelt konfrontiert sind, einer Frauenquote positiver gegenüberstehen, insbesondere wenn sie als Frauen selbst potenziell Leidtragende der Situation sind (Hypothese 2).

\subsection{Politische Orientierung}

Hinsichtlich der politischen Orientierung wird häufig zwischen den beiden Polen „links“ und „rechts“ sowie „progressiv“ und „konservativ“ unterschieden. Linksorientierte beurteilen die Chancengleichheit der Geschlechter generell deutlich kritischer und befürworten auch Veränderungen und Maßnahmen zur Geschlechterdemokratisierung stärker (Schlegel 2004). Die Ursachen für das vorhandene Ungleichgewicht sehen Linksorientierte in gesellschaftlichen Rahmenbedingungen und nicht etwa „im Biologischen“ oder „im Wesen der Geschlechter“ (ebd.,
S. 60f.). Personen mit konservativen politischen Werthaltungen orientieren sich an traditionellen Normen und Werten. Auch ist das individuelle Leistungsprinzip für sie wichtiger als Aspekte sozialer Gerechtigkeit. Somit gibt es zwei Ansatzpunkte, die - bezogen auf die Einstellung zur Frauenquote - Unterschiede hinsichtlich Personen mit progressiver vs. konservativer politischer Orientierung annehmen lassen. Konservative sollten die Frauenquote stärker ablehnen (Hypothese 3a), weil sie die traditionelle Frauenrolle in der Mutterrolle sehen und eine Änderung dessen im schlimmsten Fall zum Verlust der traditionellen Familie führen könnte. Des Weiteren zielt das individuelle Leistungsprinzip darauf $a b$, dass eine leistungsstarke Person aus eigenen Stücken in der Lage sein sollte, ihre Ziele zu erreichen. Dementsprechend wäre nicht einzusehen, warum man das Leistungsprinzip durch eine Quote aufgeben sollte, nur um „schwächeren“ Personen den Aufstieg in hohe Positionen zu ermöglichen. Weiterhin ist anzunehmen, dass konservative Männer diese Positionen in stärkerem Maße vertreten sollten, da sie sowohl vom Verlust der traditionellen Familie als auch von einer potenziellen „Unterwanderung“ des Leistungsprinzips - im Vergleich zu konservativen Frauen - nachteilig betroffen wären (Hypothese 3b).

\subsection{Wahrgenommene Chancenungleichheit}

Personen, die ein Ungleichgewicht hinsichtlich der Karrierechancen von Frauen und Männern wahrnehmen, sollten sich im Sinne der Gerechtigkeit eher für eine Frauenquote aussprechen (Hypothese 4a). Dies sollte insbesondere dann der Fall sein, wenn sie selbst von diesen geringeren Chancen betroffen sind. Es fand sich beispielsweise eine größere Wahrnehmung von Chancengleichheit der Geschlechter in unserer Gesellschaft bei Männern (61 \% Zustimmung) im Vergleich zu Frauen (36\% Zustimmung) (Schlegel 2004). Es wird dementsprechend eine Interaktion mit dem Geschlecht postuliert (Hypothese 4b).

\subsection{Erfolgsattributionen}

Betrachtet man seine oder die Erfolge anderer Personen, so kommen für deren Erlangen verschiedene Attributionen infrage. Erfolge können durch Zufall oder glückliche Umstände zustande gekommen sein. Aber auch eigenes Zutun durch Fleiß und Anstrengung oder in der Person verortete Begabungen und besondere Fähigkeiten sind mögliche Ursachen. Gehen Personen davon aus, dass sie ihre Erfolge durch eigene Leistung (sei es via Anstrengung/ Fleiß oder via Fähigkeiten) erlangt haben, so sollten sie eher gegen die Einführung einer Frauenquote sein (Hypothese 5a). Auch hier kann davon ausgegangen werden, dass dies für Männer als negativ Betroffene der Frauenquote in besonderem Maße gilt. Es wird dementsprechend eine Interaktion mit dem Geschlecht postuliert (Hypothese $5 b)$. 
ABB. 1

Modell zu Einflussfaktoren auf die Einstellung zur Frauenquote

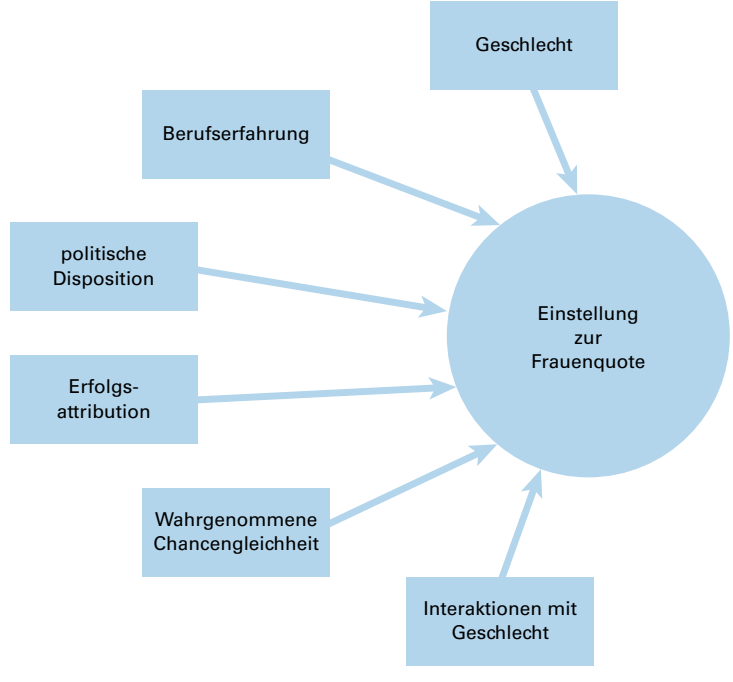

Quelle: Darstellung der Autoren

Mitteilungen

TABELLE 1

\section{Deskriptive Ergebnisse „Pro Frauenquote”}

Angaben in Prozent

\begin{tabular}{lc}
\hline & „Pro Frauenquote“ (n=412) \\
\hline Geschlecht & 34 \\
Weiblich & 14 \\
Männlich & 32 \\
\hline Berufserfahrung & 23 \\
Mitarbeitende & \\
Studierende & 18 \\
\hline Politische Orientierung & 22 \\
Konservativ (Skalenwert 1-4) & 30 \\
Mitte (Skalenwert 5-6) & \\
Progressiv (Skalenwert 7-10) & \\
\hline Erfolgsattributionen & 16 \\
Glückliche Umstände & 29 \\
$\quad$ nicht wichtig (Skalenwert 1-2) $\quad$ wichtig (Skalenwert 4-5) & \\
Fleiß/Anstrengung & $0 *$ \\
$\quad$ nicht wichtig (Skalenwert 1-2) $\quad$ wichtig (Skalenwert 4-5) & 27 \\
Begabung/Fähigkeiten & \\
$\quad$ nicht wichtig (Skalenwert 1-2) $\quad$ wichtig (Skalenwert 4-5) & 28 \\
\hline Wahrgenommene Chancenungleichheit & 26 \\
Ja & \\
Nein & 35 \\
\hline
\end{tabular}

Anmerkungen: Politische Orientierung (1 - konservativ und 10 - progressiv); Erfolgsattributionen (1 - "gar nicht wichtig" bis $5-$ "sehr wichtig“).

* Insgesamt nur 18 Personen, die angeben, Fleiß und Anstrengung wären nicht wichtig für ihren Erfolg

Quelle: Berechnungen der Autoren.

\section{Methodik und Ergebnisse}

\subsection{Stichprobe und deskriptive Ergebnisse}

Zur Prüfung der Hypothesen wurden im Juni/Juli 2012 Mitarbeitende und Studierende der Hochschule Heilbronn über den internen E-Mail-Verteiler online befragt. Die Stichprobe umfasst 412 Personen, davon $26 \%(n=105)$ Mitarbeitende (Angestellte und Beamte) und $73 \%(n=298)$ Studierende der Hochschule. ${ }^{1}$ Die Mitarbeitenden der Hochschule sind im Mittel 41 Jahre alt (SD=11.3), 59\% sind weiblich, $39 \%$ männlich. Die meisten haben ein abgeschlossenes Studium (höchster Bildungsabschluss: $82 \%$ Studium, $8 \%$ Abitur/Fachhochschulreife, $9 \%$ Realschulabschluss). Die Studierenden sind im Mittel 24 Jahre alt ( $\mathrm{SD}=2.8$ ), zu $55 \%$ weiblich und zu $45 \%$ männlich. Hinsichtlich ihrer politischen Orientierung $(\mathrm{M}=5.99 ; \mathrm{SD}=1.93)$ sind $19 \%$ der Befragten als konservativ zu kategorisieren (Skalenwert 1-4), $31 \%$ können der Mitte zugeordnet werden (Skalenwert 5-6) und 37\% (Skalenwert 7-10) der progressiven Seite. $24 \%$ der Befragten gehen von einer Chancengleichheit der Geschlechter bei Beförderungen aus (vs. $56 \%$ keine Chancengleichheit). ${ }^{2}$ Die Teilnehmenden attribuieren auf fünfstufigen Likertskalen $(1-$ „gar nicht wichtig“ bis 5 - „sehr wichtig“) im Mittel ihren Erfolg mit $\mathrm{M}=3.61$ ( $\mathrm{SD}=1.14$ ) auf glückliche Umstände, mit $\mathrm{M}=4.18$ $(\mathrm{SD}=0.80)$ auf Fleiß/Anstrengung und mit $\mathrm{M}=3.93$ $(\mathrm{SD}=0.82)$ auf Begabung/besondere Fähigkeiten. In Tabelle 1 sind die deskriptiven Ergebnisse zur Befürwortung einer Frauenquote in den verschiedenen Gruppen im Überblick dargestellt.

\subsection{Relevante Einflussfaktoren}

Um im nächsten Schritt die relevanten Einflussfaktoren zu identifizieren, wurde eine binäre logistische Regressionsanalyse (Einschluss) berechnet. Hierzu gingen - entsprechend der Hypothesen zu Interaktionen der Prädiktoren mit dem Geschlecht - Interaktionsterme (Produkt aus Prädiktor und Moderator, vgl. Aiken/West 1991) in die Analyse ein. ${ }^{3}$ Fehlende Werte wurden durch den je-

1 Für ihre Mitwirkung danken wir den beteiligten Studierenden der Hochschule Heilbronn, ohne die diese Studie nicht möglich gewesen wäre.

2 Differenzen zu $100 \%$ sind auf fehlende Angaben zurückzuführen.

3 Vor Bildung der Interaktionsterme wurden die Variablen am jeweiligen Mittelwert zentriert. Multikollinearität zwischen unabhängigen Variablen und ihrem Interaktionsterm kann das Ergebnis der Regression verfälschen. Durch Zentrieren der unabhängigen Variablen vor Bildung des Interaktions- 
TABELLE 2

\section{Faktoren für die Einstellung zur Frauenquote}

Logistische Regressionsanalyse

\begin{tabular}{|c|c|c|c|c|}
\hline \multirow[b]{2}{*}{$\begin{array}{l}\text { Prädiktoren } \\
\left(\mathrm{n}=412 ; \mathrm{df}=1 ; \text { Nagelkerkes } \mathrm{R}^{2}=.244\right)\end{array}$} & \multicolumn{4}{|c|}{ Kriterium „PRO Frauenquote” } \\
\hline & $B$ & $S E$ & Wald & $\operatorname{Exp}(B)$ \\
\hline Frauen & 1.07 & 0.36 & $8.79 * *$ & 2.93 \\
\hline Berufserfahrung & 0.17 & 0.33 & 0.27 & 1.19 \\
\hline Polit. Orientierung konservativ-progressiv & 0.24 & 0.08 & $8.06 * *$ & 1.27 \\
\hline Wahrgenommene Chancenungleichheit & 1.89 & 0.47 & $16.41 * * *$ & 6.63 \\
\hline Erfolgsattribution Glück & 0.38 & 0.13 & $8.76 * *$ & 1.46 \\
\hline Erfolgsattribution Fleiß/Anstrengung & 0.46 & 0.20 & $5.27 *$ & 1.58 \\
\hline Erfolgsattribution Begabung/Fähigkeiten & 0.17 & 0.18 & 0.95 & 1.19 \\
\hline Frauen $\times$ Berufserfahrung & 1.22 & 0.71 & $2.99^{\top}$ & 3.39 \\
\hline Frauen $\mathrm{x}$ polit. Orientierung & -0.41 & 0.18 & $5.28 *$ & 0.67 \\
\hline Frauen $x$ wahrg. Chancenungleichheit & -1.56 & 0.99 & $2.49^{\top}$ & 0.21 \\
\hline Frauen x Erfolgsattr. Glück & -0.05 & 0.27 & 0.03 & 0.95 \\
\hline Frauen 'x Erfolgsattr. Fleiß/Anstrengung & -0.39 & 0.43 & 0.85 & 0.68 \\
\hline Frauen x Erfolgsattr. Begabung/Fähigkeiten & -0.23 & 0.38 & 0.36 & 0.80 \\
\hline Konstante & -1.45 & 0.17 & $73.68 * * *$ & 0.23 \\
\hline
\end{tabular}

Anmerkungen: *** $\mathrm{p} \leq .001 ;{ }^{*} \mathrm{p} \leq .01 ;{ }^{*} \mathrm{p} \leq .05 ;{ }^{\top} \mathrm{p} \leq .15$

Die resultierende Regressionsgleichung lautet:

$y=-1.45+1.07 x_{1}+0.17 x_{2}+0.24 x_{3}+1.89 x_{4}+0.38 x_{5}+0.46 x_{6}+0.17 x_{7}+1.22 x_{1} x_{2}$

$-0.41 x_{1} x_{3}-1.56 x_{1} x_{4}-0.05 x_{1} x_{5}-0.39 x_{1} x_{6}-0.23 x_{1} x_{7}$

wobei $x_{1}=$ Frau (1, Mann = 0$) ; x_{2}=$ Berufserfahrung/Mitarbeitende $(1$, keine Berufserfahrung/Studierende $=0) ; x_{3}=$ polit. Orientierung $(1=$ konservativ bis

$10=$ progressiv); $x_{4}=$ wahrg. Chancenungleichheit $(1 ;$ nein $=0) ; x_{5}=$ Erfolgsattribution Glück; $x_{6}=$ Erfolgsattribution Fleiß/Anstrengung; $x_{7}=$ Erfolgsattribution

Begabung/besondere Fähigkeiten ( $x_{5}$ bis $x_{7}: 1=$ "gar nicht wichtig“ bis $5=$ "sehr wichtig“).

Quelle: Berechnungen der Autoren.

weiligen Mittelwert ersetzt. Auf Basis der Regressionsanalyse werden insgesamt 77,5\% der Personen richtig klassifiziert.

Wie in Tabelle $2 \mathrm{zu}$ sehen, haben Geschlecht $(B=1.07$; $p=.003)$, politische Orientierung $(B=0.24 ; p=.005)$, wahrgenommene Chancenungleichheit $(B=1.89 ; p=.000)$, Erfolgsattribution Glück $(B=0.38 ; p=.003)$, Erfolgsattribution Fleiß/Anstrengung $(B=0.46 ; p=.022)$ sowie die Interaktionsterme Geschlecht $\times$ Berufserfahrung $(B=1.22$; $p=.084)$, Geschlecht $\times$ politische Orientierung $(B=-0.41$; $p=.022)$ und Geschlecht $\times$ wahrgenommene Chancenungleichheit $(B=-1.56 ; p=.115)$ einen (tendenziell) signifikanten Einfluss. Die anderen Prädiktoren haben keinen Einfluss auf die Einstellung zur Frauenquote. Somit sind Frauen, Progressive und Personen, welche eine Chancenungleichheit der Geschlechter wahrnehmen, häufiger für die Frauenquote. Zudem wird die Frauenquote vermehrt von Personen befürwortet, die ihren eigenen Erfolg auf Glück sowie Fleiß und Anstrengung zurückführen.

Die tendenzielle Interaktion „Geschlecht $\times$ Berufserfahrung" (Hypothese 2) zeigt, dass Frauen mit Berufserfahrung (Mitarbeiterinnen, $43 \%$ ) im Vergleich zu Frauen ohne Berufserfahrung (Studenteninnen, 30\%) die Frauenquote stärker befürworten, während sich Männer mit (Mitarbeiter, $15 \%$ ) und ohne Berufserfahrung (Studenten, 13\%) nicht unterscheiden (Abbildung 2). Die Interaktion „Ge- schlecht $\times$ politische Orientierung“" (Hypothese $3 b$ ) zeigt, dass sich progressive (34\%) und konservative Frauen (33\%) hinsichtlich der Befürwortung der Quote nicht unterscheiden, während bei Männern die politische Orientierung erheblichen Einfluss hat. ${ }^{4}$ Konservative Männer (3\%) befürworten die Quote deutlich weniger als progressive (30\%, Abbildung 3). Des Weiteren ist die Interaktion „Geschlecht $\times$ wahrgenommene Chancenungleichheit“ (Hypothese $4 b$ ) tendenziell signifikant. Frauen und Männer, die Chancenungleichheit wahrnehmen, befürworten die Quote häufiger, bei Frauen ist dieser Unterschied etwas stärker ausgeprägt (Frauen: $37 \%$ vs. $17 \%$; Männer $19 \%$ vs. $3 \%$ ).

terms kann die Multikollinearität reduziert werden. Obwohl es sich beim Zentrieren am Mittelwert um eine rein lineare Transformation handelt, kann dies einen erheblichen Effekt auf Regressionskoeffizienten in Regressionsanalysen mit Interaktionstermen haben (Aiken/West 1991). Dies liegt daran, dass die Kovarianz zwischen dem Interaktionsterm und den einzelnen Komponenten in Teilen vom Mittelwert der einzelnen Komponenten abhängt. Zentrieren ändert den Mittelwert und damit die Kovarianz (ebd.).

4 Für die Berechnung der gemittelten vorhergesagten Wahrscheinlichkeiten wurden für Konservative die Skalenwerte 1-4 und für Progressive die Skalenwerte 7-10 auf der Skala politische Orientierung eingesetzt. 
ABB. 2

\section{Interaktion Geschlecht x Berufserfahrung, vorhergesagte Wahrscheinlichkeiten pro Frauenquote}

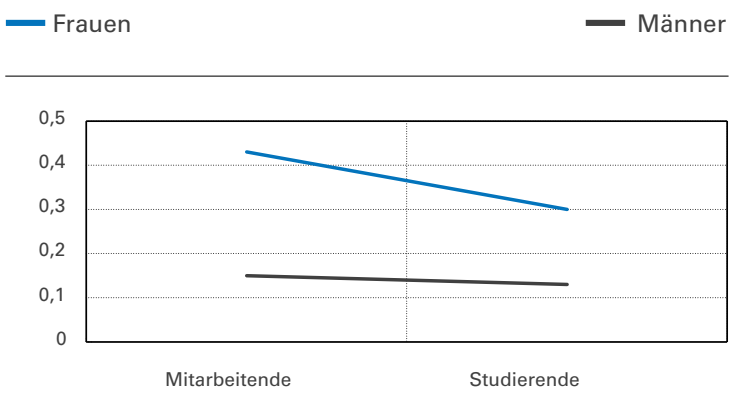

\section{Diskussion}

Wie bereits mehrfach belegt (z. B. Wagner/Schmermund 2000; Krings et al. 2007; Schlegel 2004; Wippermann 2010), so weist auch die vorliegende Studie einen Einfluss des Geschlechts auf die Einstellungen zur Frauenquote aus: Frauen stehen der Gleichstellungsmaßnahme „Frauenquote“ positiver gegenüber als Männer. Als Betroffene des aktu-

ABB. 3

\section{Interaktion Geschlecht x politische Orientierung, vorhergesagte Wahrscheinlichkeiten pro Frauenquote

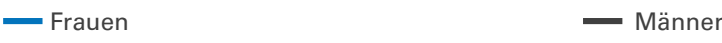

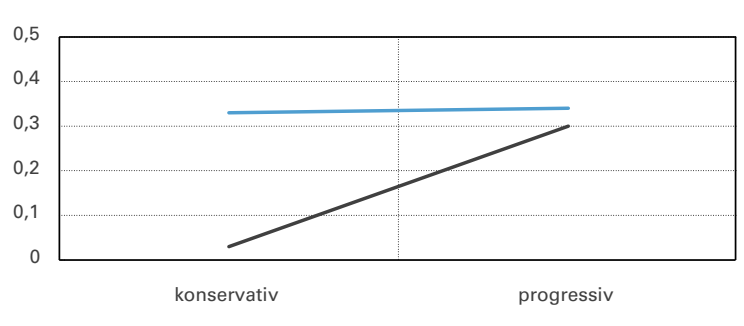

Quelle: Darstellung der Autoren

ellen Ungleichgewichts und Profitierende einer Quote ist dies zu erwarten. Dies ist für bestimmte Subgruppen besonders deutlich: So sehen beispielsweise konservative Männer und Männer, die keine Chancenungleichheit im Berufsleben wahrnehmen, fast keinen Anlass für eine Quo- te. Bei den Frauen ist die größte Befürwortung bei jenen mit Berufserfahrung zu finden.

Der erwartete Einfluss der Berufserfahrung zeigt sich allerdings nicht per se. Jedoch weist die tendenzielle Interaktion mit dem Geschlecht den postulierten Effekt für Frauen nach. Während sich Männer mit und ohne Berufserfahrung nicht unterscheiden, favorisieren Frauen mit Berufserfahrung die Einführung einer Quote stärker als Frauen ohne Berufserfahrung. Da die Bedingungen für beide Geschlechter in Schule und Studium noch vergleichbar sind, scheinen Frauen, die noch nicht im Berufsleben angekommen sind, noch stärker an die Potenziale von Bildung und Leistung zu glauben. Die direkten oder indirekten Erfahrungen mit ungleichen Job- und Karrierechancen, mit denen Frauen im Berufsleben konfrontiert sind, scheinen dementsprechend dazu zu führen, dass eine Frauenquote für die Auflösung gesellschaftlicher Änderungen als notwendig erachtet wird. Um diese Effekte genauer zu untersuchen, sollte Berufserfahrung auch auf anderen Wegen operationalisiert werden, beispielsweise über die Berufserfahrung in Jahren oder anhand inhaltlich-qualitativer Begebenheiten wie einschneidende Erlebnisse in Bewerbungssituationen oder bei potenziellen oder erlebten Beförderungssituationen.

Die politische Einfärbung der Diskussion um die Frauenquote zeigt sich differenziert auch in den Befunden der vorliegenden Studie zum Einfluss der politischen Orientierung: Je progressiver die politische Selbsteinschätzung, desto größer ist die Unterstützung der Quote. Die Interaktion mit dem Geschlecht zeigt jedoch, dass dieser Sachverhalt ausschließlich von Männern dominiert ist. Während Frauen über unterschiedliche politische Orientierungen hinweg in gleichem Maße für die Frauenquote votieren, lassen sich unter konservativen Männern praktisch keine Befürworter finden. Progressive Männer hingegen heißen eine Frauenquote deutlich häufiger gut. Dies ist, wie oben ausgeführt, z. B. erklärbar über die potenzielle Gefährdung der traditionellen Frauenrolle durch die Einführung von Quoten. Auch die mit Konservatismus einhergehende Höhergewichtung des individuellen Leistungsprinzips kontra eines Protegierens Schwächerer um sozialer Gerechtigkeit willen, kann diesen Einstellungsunterschied konservativer und progressiver Männer verursachen.

Bei Frauen scheinen diese Überzeugungen keinen Einfluss auf die wahrgenommene Notwendigkeit einer Quote zu haben. Dass sich konservative wie progressive Frauen gleichermaßen für die Einführung einer Quote aussprechen, ist beispielsweise erklärbar durch die von beiden Seiten geteilte Wahrnehmung, dass die gesellschaftlichen Rahmenbedingungen gerade nicht oder nicht unbedingt das Leistungsprinzip unterstützen. Diese Sichtweise werden negativ Betroffene (Frauen) eher einnehmen als positiv Betroffene (Männer). Eine Frau, die trotz Leistung nicht nach „oben“ kommt, wird dies als nicht berechtigte Benachteiligung und als Verstoß gegen das Leistungsprinzip wahrnehmen. Ein Mann, der durch Leistung 
aufsteigt, kann darin die Bestätigung des individuellen Leistungsprinzips sehen.

Evident ist der Einfluss wahrgenommener Chancenungleichheit: Personen, die ein Ungleichgewicht hinsichtlich der Karrierechancen von Frauen und Männern wahrnehmen, begrüßen - im Sinne der Gerechtigkeit - eine Frauenquote stärker. Die tendenzielle Interaktion mit dem Geschlecht zeigt, dass dies, wie angenommen, besonders für Frauen der Fall ist, da sie vielfach häufiger als Männer von den geringeren Chancen betroffen sind.

Aufschlussreich ist schließlich auch der Einfluss von Erfolgsattributionen: Personen, die ihren Erfolg auf Glück zurückführen, befürworten die Quote in stärkerem Maße. Wenn jemand Glück für das Erreichen seiner persönlichen und beruflichen Ziele verantwortlich macht, so hätte es auch ebenso anders kommen können; Gerechtigkeit spielt hier keine Rolle. Eine Frauenquote könnte dafür sorgen, dieses „pure Glück“ einzuschränken, was wiederum die verstärkte Befürwortung einer Quote erklären kann. Allerdings sprechen sich auch Personen, die ihren Erfolg auf Fleiß und Anstrengung zurückführen, häufiger für eine Frauenquote aus. Dies kann dadurch erklärbar sein, dass zwar der eigene Fleiß als Karrieremacher angesehen wird, dass aber gleichzeitig beobachtet wird, dass trotz Fleiß und Anstrengung Karriereziele nicht erreicht werden. Was wiederum eine unfaire Situation darstellt, die - z. B. mittels einer Quote - reguliert werden sollte.

Zusammenfassend lässt sich feststellen, dass (eigene) Betroffenheit in verschiedensten Ausprägungen (hier beispielsweise erkennbar über Geschlecht, wahrgenommene Chancen(un)gleichheit und Berufserfahrung) ein relevanter Faktor für die Einstellung zur Frauenquote zu sein scheint. Eine Politik, welche die gesellschaftliche Akzeptanz für Gleich stellungsmaßnahmen wie die Frauenquote erhöhen möchte, sollte sich dieser Einflussfaktoren bewusst sein.

\section{Fazit und Ausblick}

Die vorliegende Analyse liefert Indikatoren für Einflussfaktoren auf berufliche Gleichstellungsmaßnahmen wie die Einführung einer Frauenquote.

Einschränkend anzumerken ist die Zusammensetzung der Stichprobe aus Personen mit höheren Bildungsabschlüssen, deren Realität im Arbeitsalltag sich von anderen Berufen unterscheidet (Hochschulbeschäftigte) oder voraussichtlich unterscheiden wird (Studierende). Wichtig wäre es somit, verschiedene Branchen und eine größere Spreizung hinsichtlich Ausbildungsabschlüssen gegebenenfalls mit Berücksichtigung typischer Charakteristika der Arbeitsplätze (wie z. B. Handlungsspielraum, Ausmaß an Eigenverantwortung etc.) in Folgeanalysen einzubeziehen.

Der Einfluss politischer Sozialisierung zeigte sich vor allem bei Männern. Die Haltung zur Frauenquote ist in der öffentlichen Diskussion und Wahrnehmung auch eng mit Parteien verknüpft. Weitere Forschung sollte die Parteinähe berücksichtigen. Ebenfalls von Interesse wären konkrete Erfahrungen im Berufsleben. Wenn auch Erkenntnisse über verschiedene Einflussfaktoren gewonnen wurden, so stellt sich trotzdem die Frage, warum der Anteil der Befürworter einer Frauenquote so gering ist. Die Vergangenheit hat gezeigt, dass es zur Abschaffung von Ungleichheit und systematischer Diskriminierung gesetzlicher Rahmenbedingungen bedarf (historische Beispiele sind der Zugang zum Studium für Frauen und das Wahlrecht für Frauen). Weitere Forschung sollte sich deshalb auch den Vorurteilen gegenüber der „Quotenfrau“ und dem Mythos, bei Karriere zähle ausschließlich Leistung, annehmen. Dieser Mythos bildet nicht die Realität ab und lässt Frauen zudem mit dem Gefühl zurück, eine ggf. weniger erfolgreiche Karriere selbst verschuldet zu haben oder eben im umgekehrten Fall - ohne ausreichende eigene Leistung als Quotenfrau vor eigentlich besseren Männern bevorzugt worden zu sein. Vor diesem Hintergrund ist nachvollziehbar, was vielen, auch vielen Frauen, ein klares "Ja“ zu einer Quote, die bestehende Ungleichbehandlung abbauen könnte, erschwert. Wenn man jedoch nicht nur bestimmte Frauen fördern möchte und es nicht nur um das Top-Management und Aufsichtsräte geht, ist auch der Blick auf die Lebenswelt und insbesondere auf die institutionell nach wie vor für beide Geschlechter erschwerten Möglichkeiten, Familie und Beruf miteinander vereinbaren zu können, auszuweiten. Unter Status-quoBedingungen können Frauen, die nach wie vor das „Unvereinbarkeitsproblem“ individuell zu lösen versuchen, nicht in ausreichender Zahl in die mittleren Führungsebenen vordringen. Dies ist ein weiterer Aspekt, der die Einstellung zur Frauenquote beeinflussen könnte. Immer wieder wird in diesem Kontext auch argumentiert, die meisten Frauen wollten gar keine Karriere machen und ihre Interessen lägen in anderen Bereichen. Dies mag für einen Teil der Frauen (wie auch Männer) zutreffen, doch sicherlich nicht für alle, womit das bestehende Ungleichgewicht in der Besetzung von Führungspositionen wieder zutage tritt. Weitere Forschung sollte Themen wie eigene Karriereziele, den Stellenwert von Familie/Zeit für die Familie, die Existenz eigener Kinder, die wahrgenommene Vereinbarkeit von Beruf und Familie etc. mit berücksichtigen.

\section{LITERATUR}

Aiken, L. S./West, S.G. (1991): Multiple regression: Testing and interpreting interactions, Newbury Park, CA

Bundesministerium für Familie, Senioren, Frauen und Jugend (BMFSFJ) (2012a): Flexi-Quote und Stufenplan „Frauen und Männer in Führungspositionen“, 24.07., www.bmfsfj.de/BMFSFJ/Service/themen-lotse,did=172756.html (letzter Zugriff: 18.12.2012)

Bundesministerium für Familie, Senioren, Frauen und Jugend (BMFSFJ)

(2012b): Wirtschaft übergibt Kristina Schröder Statusbericht zu Frauen in Führungspositionen, 28.06., www.bmfsfj.de/BMFSFJ/gleichstellung,did=187194. html (letzter Zugriff: 02.10.2014)

Bundesministerium für Familie, Senioren, Frauen und Jugend (BMFSFJ)

(2012c): Ziele der DAX 30 Aufsichtsräte, www.flexi-quote.de/ziele-der-dax30-aufsichtsraete.html (letzter Zugriff 18.12.2012)

Bundesministerium für Familie, Senioren, Frauen und Jugend (BMFSFJ)

(2014): Gesetz für die gleichberechtigte Teilhabe von Frauen und Männern an Führungspositionen in der Privatwirtschaft und im Öffentlichen Dienst, www. bmfsfj.de/BMFSFJ/gleichstellung,did=205630.html (Letzter Zugriff: 01.10.2014) Bundesregierung (2011): Regierungspressekonferenz vom 2. Februar, www. bundesregierung.de/Content/DE/Mitschrift/Pressekonferenzen/2011/02/2011-0202-regpk.html (letzter Zugriff: 13.12.2012)

Destatis (2011): Hochschulen auf einen Blick 2011, Statistisches Bundesamt Wiesbaden, www.destatis.de/DE/Publikationen/Thematisch/BildungForschungKultur/Hochschu-len/BroschuereHochschulenBlick0110010117004.pdf? blob=publicationFile (letzter Zugriff: 26.11.2012)

DAPD (2011): Frauen in Führungspositionen: Merkel befürwortet „Flexi-Quote", www.handelsblatt.com/politik/deutschland/frauen-in-fuehrungspositionen-merkel-befuerwortet-flexi-quote/5501396.html (letzter Zugriff 02.10.2014)

Deutscher Corporate Governance Kodex (2012): Deutscher Corporate Governance Kodex, 15.05.2012, www.corporate-governance-code.de/ger/download/ kodex_2012/D_CorGov_Endfassung_Mai_2012.pdf (letzter Zugriff 18.12.2012) 
Deutsches Institut für Wirtschaftsforschung (DIW) Berlin (2014): Pressemitteilung vom 17.07.: Frauenanteil in DAX 30 Vorständen weiter gesunken, www. diw.de/de/diw_01.c.470215.de/themen_nachrichten/frauenanteil_in_dax_30_ vorstaenden_weiter_gesunken.html (letzter Zugriff: 01.10.2014)

Endres, H. (2010): Frauenfreundliches Europa, www.manager-magazin.de/unternehmen/artikel/0,2828,704800,00.html (letzter Zugriff: 02.10.2014)

Europäische Kommission (2012): Gleichstellung von Frauen und Männern, http://ec.europa.eu/justice/gender-equality/gender-pay-gap/national-situation/ index_de.htm\#h2-10 (letzter Zugriff: 02.10.2014)

Europäisches Parlament (2013): 40 \% der Sitze in Aufsichtsräten für Frauen, http://www.europarl.europa.eu/news/de/news-room/content/20131118IPR25532/ html/40-der-Sitze-in-Aufsichtsr\%C3\%A4ten-f\%C3\%BCr-Frauen (letzter Zugriff 26.01.2015).

Eurostat (2010): Geschlechtsspezifischer Lohnunterschied ohne Anpassungen, http://epp.eurostat.ec.europa.eu/tgm/refreshTableAction.do?tab=table\&plugin =1\&pcode=tsdsc340\&language=de (letzter Zugriff: 10.12.2012)

Französische Botschaft Berlin (2011): Das Gesetz zur Frauenquote in Aufsichtsräten von Unternehmen in Frankreich, www.ambafrance-de.org/Das-Gesetzzur-Frauenquote-in (letzter Zugriff: 15.01.2013)

Germany Trade and Invest (2012): Lohn- und Lohnnebenkosten - Italien, www.gtai.de/GTAI/Navigation/DE/Trade/maerkte,did=727532.html (letzter Zugriff 15.01.2013)

Hausmann, R./Bekhouche, Y./Tyson, L. D. /Zahidi, S. (2013): The global gender gap report 2013, World Economic Forum, www3.weforum.org/docs/WEF_ GenderGap_Report_2013.pdf (letzter Zugriff: 01.10.2014)

Holst, E./Busch, A./Kröger, L. (2012): Führungskräfte-Monitor 2012: DIW-Berlin, Politikberatung kompakt (65)

Holst, E./Kirsch, A. (2014): Frauen sind in Vorständen großer Unternehmen in Deutschland noch immer die Ausnahme - moderat steigende Anteile in Aufsichtsräten, in: DIW Wochenbericht 81 (3), S. 19-31

Kienbaum (2012): Kienbaum veröffentlicht Analyse zu weiblichen Aufsichtsräten im Dax-30, www.kienbaum.de/desktopdefault.aspx/tabid-501/649_read13743 (letzter Zugriff: 14.01.2013)

König, E. (2010): Stufenplan zur 40-Prozent-Frauenquote, www.euractiv.de/un ternehmen-und-arbeit/artikel/stufenplan-zur-40-prozent-frauenquote-002853 (letzter Zugriff: 02.10.2014)

Krings, F./Tschan, F./Bettex, S. (2007): Determinants of attitudes toward affirmative action in a Swiss sample, in: Journal of Business and Psychology 21 (4), S. 585-611

Lindecke, C. (2005): Von der Gleichstellung der Geschlechter zur nachhaltigen Familienpolitik, in: WSI-Mitteilungen 58 (8), S. 473-477, http://www.boeckler. de/wsi-mitteilungen_24593 24598.htm

Mischke, J./Wingerter, C. (2012): Frauen und Männer auf dem Arbeitsmarkt, Statistisches Bundesamt Wiesbaden, www.destatis.de/DE/Publikationen/Thematisch/Arbeitsmarkt/Erwerbstaetige/BroeschuereFrauenMaennerArbeitsmarkt0010018129004.pdf?_blob=publicationFile (letzter Zugriff: 02.10.2014) Sattelberger, T. (2011): Praxisbeispiel Telekom: Die Frauenquote - Qual der Entscheidung und der schwierige Weg vor uns, in: Krell, G. (Hrsg.): Chancengleichheit durch Personalpolitik, Wiesbaden, S. 429-435
Schlegel, U. (2004): Akzeptanz von Frauenfördermaßnahmen und Gender Mainstreaming - am Beispiel einer Studierendenbefragung an Fachhochschulen in Sachsen-Anhalt, in: Wüst, H. (Hrsg.): Gender Konkret! Chancengleicheit von Frauen an Fachhochschulen - Dokumentation der Fachtagung 2003, Berlin, S. 55-80

Sozialdemokratische Partei Deutschlands (SPD) (2012): Frauenquote - EU-Pläne stocken, www.spd.de/mobilespd/news/\%3Bjsessionid=832A61E48CC62381 7936B736DC09F7DF?mid=79842 (letzter Zugriff: 02.10.2014)

Storvik, A./Teigen, M. (2010): Das norwegische Experiment - eine Frauenquote für Aufsichtsräte, www.amb-norwegen.ch/Global/SiteFolders/webber/Das \%20 norwegische\%20Experiment.pdf (letzter Zugriff: 02.10.2014)

Tomasson, R. F./Crosby, F. J./Herzberger, S.D. (1996): Affirmative action, the pros and cons of policy and practice, Washington DC

Wagner, U./Schmermund, A. (2000): Einstellungen zu Maßnahmen der beruflichen Gleichstellung, in: Zeitschrift für Arbeits- und Organisationspsychologie 44 (2), S. 83-89

Weinmann J. (2010): Frauen und Männer in verschiedenen Lebensphasen, Statistisches Bundesamt Wiesbaden, www.destatis.de/DE/Publikationen/Thematisch/Bevoelkerung/HaushalteMikrozensus/BroschuereFrauenMaenner0010013109001.pdf?__blob=publicationFile

Wippermann, C. (2010): Frauen in Führungspositionen - Barrieren und Brücken, Bundesministerum für Familie, Senioren, Frauen und Jugend, www. bmfsfj.de/RedaktionBMFSFJ/Broschuerenstelle/Pdf-Anlagen/frauen-in-f_C3 BChrungspositionen-deutsch, property $=$ pdf, bereich $=$ bmfsfj, sprache $=d e, r w b=t r$ ue.pdf (lezter Zugriff: 02.10.2014)

\section{AUTOREN}

FRANZISKA DRESCHER, Dr., leitet das Heilbronner Institut für angewandte Marktforschung an der Hochschule Heilbronn. Arbeitsschwerpunkte: Akzeptanz- und Einstellungsforschung, sozialwissenschaftliche Evaluation, Kundenforschung, Produktforschung, User Experience.

franziska.drescher@hs-heilbronn.de

PETER SCHROTT, Prof., PhD, ist Direktor des Heilbronner Instituts für angewandte Marktforschung an der Hochschule Heilbronn. Arbeitsschwerpunkte: Meinungsforschung, Wahlforschung und politische Kommunikation, Forschungsmethoden, Makroökonomie und Politik.

peter.schrott@hs-heilbronn.de 